Research Article

\title{
On the Formal Integrability Problem for Planar Differential Systems
}

\author{
Antonio Algaba, ${ }^{1}$ Cristóbal García, ${ }^{1}$ and Jaume Giné ${ }^{2}$ \\ ${ }^{1}$ Departamento de Matemàticas, Facultad de Ciencias, Avda. Tres de Marzo s/n, 21071 Huelva, Spain \\ ${ }^{2}$ Departament de Matemàtica, Universitat de Lleida, Avda. Jaume II, 69, 25001 Lleida, Catalonia, Spain
}

Correspondence should be addressed to Jaume Giné; gine@matematica.udl.cat

Received 15 November 2012; Accepted 28 January 2013

Academic Editor: Sung Guen Kim

Copyright (C) 2013 Antonio Algaba et al. This is an open access article distributed under the Creative Commons Attribution License, which permits unrestricted use, distribution, and reproduction in any medium, provided the original work is properly cited.

\begin{abstract}
We study the analytic integrability problem through the formal integrability problem and we show its connection, in some cases, with the existence of invariant analytic (sometimes algebraic) curves. From the results obtained, we consider some families of analytic differential systems in $\mathbb{C}^{2}$, and imposing the formal integrability we find resonant centers obviating the computation of some necessary conditions.
\end{abstract}

\section{Introduction}

One of the main open problems in the qualitative theory of differential systems in $\mathbb{R}^{2}$ is the distinction between a center and a focus, called the center problem, and its relation with the integrability problem; see, for instance, [1-5]. The notion of center can be extended to the case of a $p$ : $-q$ resonant singular point of a polynomial vector field in $\mathbb{C}^{2}$ and to some other situations (resonant node, saddle node, and nonelementary singular points); see [6]. Recently, several works about this subject have appeared where the classification of the resonant centers for certain families is given using powerful computational facilities; see, for instance, [6-17].

There exist several methods to find necessary conditions; see $[1,18]$. However, there is no general method to provide the sufficiency for each family that satisfies some necessary conditions. The sufficiency is obtained verifying that the system is Hamiltonian, or that it has certain reversibility, or certain Lie symmetry, or finding a first integral well defined in a neighborhood of the singular point, sometimes finding an integrating factor that allows to construct a first integral, reducing the system to an integrable system; see, for instance, $[4,5,18-23]$ and the references therein. These methods have proved ineffective in certain families that already verify some necessary conditions, and in many papers some cases are established as open problems; see, for instance, [7, 10, 11]. Carrying, in practice, to prove the sufficiency by ad hoc methods for each particular case where it has been possible, see $[6-8,12,16,17,24-26]$. One of the ways to prove the sufficiency is proving the existence of a formal first integral. The existence of a formal first integral implies the existence of an analytic first integral for an isolated singularity from the results obtained by Mattei and Moussu; see [27] and Theorem 2 in Section 2.

Therefore, one of the main objectives for the next years will be to find an algorithm (if exists) or methods that give directly sufficient conditions to decide whether or not a differential system in the plane admits a formal first integral at a singular point.

This paper is the first step in this direction. In this paper, we study the analytic integrability through the formal integrability and we show its connection, in some cases, with the existence of invariant analytic (sometimes algebraic) curves. Moreover, from the results obtained in this work, we consider some families of analytic differential systems in $\mathbb{C}^{2}$, and imposing the formal integrability we find centers or 
resonant centers obviating the computation of the necessary conditions (see, for instance, Proposition 11).

\section{Definitions and Preliminary Concepts}

We first consider a real system of ordinary differential equations on $\mathbb{R}^{2}$ with an isolated singular point at the origin, whose linear parts are nonzero pure imaginary numbers. By a linear change of coordinates and a constant time rescaling, the system takes the form

$$
\dot{u}=v+\cdots, \quad \dot{v}=-u+\cdots .
$$

The classical Poincaré-Liapunov center theorem states that the origin is a center if and only if the system admits an analytic first integral of the form $\phi(u, v)=u^{2}+v^{2}+\cdots$; see, for instance, $[18,28-31]$ and the references therein.

If system (1) is complexified in a natural way taking $z=u+i v$, then we obtain a differential equation of the form $\dot{z}=i z+\cdots$. In this case one constructs, step by step, the formal first integral $\Phi=z \bar{z}+\cdots$ satisfying the equation $\dot{\Phi}=v_{3}|z|^{4}+v_{5}|z|^{6}+\cdots$, where the coefficients $v_{2 i+1}$, called the focus quantities, are polynomials in the coefficients of the original system. The theorem of PoincaréLiapunov $[32,33]$ says that the point $z=0$ is a center if and only if all the $v_{2 i+1}=0$. Existence of a first integral $\phi$ is equivalent to existence of an analytic first integral for the complexified equation of the form $\Phi=z \bar{z}+\cdots$. Taking the complex conjugated equation, there arises an analytic system of ordinary differential equations on $\mathbb{C}^{2}$ of the form $\dot{z}=i z+\cdots, \dot{w}=-i w+\cdots$ where $w=u-i v$. Hence, after the complexification, the system is transformed into an analytic system with eigenvalues $+i$ and $-i$. This is the $1:-1$ resonant singular point and the numbers $v_{2 i+1}$ become the coefficients of the resonant terms in its orbital normal form. Dulac [34] chosen this way to approach the center problem for quadratic systems.

The next natural generalization is to consider the case of an analytic vector field in $\mathbb{C}^{2}$ with $p:-q$ resonant elementary singular point

$$
\dot{x}=p x+\cdots, \quad \dot{y}=-q y+\cdots,
$$

where $p, q \in \mathbb{Z}$ with $p, q>0$. These facts motivate the generalization of the concept of real center to certain classes of systems of ordinary differential equations on $\mathbb{C}^{2}$. In this case, we have the following definition of a resonant center or focus, coming from Dulac [34]; see also [6].

Definition 1. A $p:-q$ resonant elementary singular point of an analytic system is a resonant center if, and only if, there exists a local meromorphic first integral $\Phi=h_{0}+\cdots$, with $h_{0}=x^{q} y^{p}$. This singular point is a resonant focus of order $k$ if, and only if, there is a formal power series $\Phi=x^{q} y^{p}+\cdots$ with the property $\dot{\Phi}=g_{k} h_{0}^{k+1}+\cdots$.

Recently, several works are focused on the study of resonant centers for complex analytic systems. In these works, the integrability and linearizability problems are studied.
The linearizability problem is focused on the study of the existence of an analytic change of coordinates that linearize the complex analytic system.

Mattei and Moussu [27] proved the next result for all isolated singularities.

Theorem 2. Assume that system (1) or (2) with an isolated singularity at the origin has a formal first integral $H \in$ $\mathbb{R}[[x, y]]$ around it. Then, there exists an analytic first integral around the singularity.

In the light of the former result, we can conclude that in order to prove the existence of an analytic first integral we only need to prove the existence of a formal first integral. Therefore, the formal integrability problem takes a primary role for the upcoming investigations on the center and resonant center problem.

\section{Blowup of Resonant Saddles}

In this section, we will consider the first method to approach the formal integrability problem for resonant centers. We consider the resonant analytic system (2). We now do the blowup of this singularity. This means that we apply the map $(x, y) \rightarrow(x, z)=(x, y / x)$. The point $x=y=0$ is replaced by the line $x=0$, which contains two singular points that correspond to the separatrices of (2) and are the saddles: $p_{1}$ which is $(p+q):-p$ resonant and $p_{2}$ which is $(p+q):-q$ resonant. The following lemma is proved in [12] using the normal orbital form of a resonant analytic system (2).

Lemma 3. If one of the points $p_{1}$ or $p_{2}$ is orbitally analytically linearizable then the point $x=y=0$ is a $p:-q$ resonant center.

As a corollary of this lemma, we get the following useful property which can be used to approach the resonant center problem. If the point $p_{1}$ is orbitally analytically linearizable then it has a formal first integral in the variables $(x, z)$ of the form

$$
H=x^{p} z^{q+p} \sum_{n=0}^{\infty} f_{n}(x) z^{n}
$$

with $f_{n}(0) \neq 0$. Hence, the original $p:-q$ resonant point has a nonformal first integral of the form

$$
x^{-q} y^{q+p} \sum_{n=0}^{\infty} f_{n}(x) x^{-n} y^{n}=\sum_{n=0}^{\infty} f_{n}(x) x^{-q-n} y^{q+p+n} .
$$

Consequently, if the resonant point $p:-q$ has a non formal first integral of the form (4) then by Lemma 3 the resonant point $p:-q$ is a resonant center. The same result can be established for the other saddle $p_{2}$. These types of results were also given by Bruno in $[35,36]$. 


\section{The $\varepsilon$-Method for Resonant Centers}

We recall in this section the $\varepsilon$-method developed in [23] which we apply here to resonant centers. We consider system (2) which we write into the form

$$
\begin{aligned}
& \dot{x}=P(x, y)=p x+F_{1}(x, y), \\
& \dot{y}=Q(x, y)=-q y+F_{2}(x, y),
\end{aligned}
$$

where $F_{1}(x, y)$ and $F_{2}(x, y)$ are analytic functions without constant and linear terms, defined in a neighborhood of the origin.

To implement the algorithm we introduce a rescaling of the variables and a time rescaling given by $(x, y, t) \rightarrow$ $\left(\varepsilon^{\bar{p}} x, \varepsilon^{\bar{q}} y, \varepsilon^{\bar{r}} t\right)$ where $\varepsilon>0$ and $\bar{p}, \bar{q}$, and $\bar{r} \in \mathbb{Z}$ and system (5) takes the form

$$
\begin{aligned}
& \dot{x}=\varepsilon^{\bar{r}-\bar{p}}\left(p \varepsilon^{\bar{p}} x+F_{1}\left(\varepsilon^{\bar{p}} x, \varepsilon^{\bar{q}} y\right)\right), \\
& \dot{y}=\varepsilon^{\bar{r}-\bar{q}}\left(-q \varepsilon^{\bar{q}} y+F_{2}\left(\varepsilon^{\bar{p}} x, \varepsilon^{\bar{q}} y\right)\right) .
\end{aligned}
$$

We choose $\bar{p}, \bar{q}, \bar{r}$ in such a way that system (6) will be analytic in $\varepsilon$. Hence, by the classical theorem of the analytic dependence with respect to the parameters, we have that system (6) admits a first integral which can be developed in power of series of $\varepsilon$ because it is analytic with respect to this parameter. Therefore, we can propose the following development for the first integral:

$$
H(x, y)=\sum_{k=0}^{\infty} \varepsilon^{k} h_{k}(x, y),
$$

where $h_{k}(x, y)$ are arbitrary functions. We notice that $F_{1}(x, y)$ and $F_{2}(x, y)$ are analytic functions, where both can be null and $F_{1}(0,0)=F_{2}(0,0)=0$, so we can develop them, in a neighborhood of the origin, as convergent series of $x$ and $y$ of the form

$$
\begin{aligned}
& F_{1}(x, y)=p_{n}(x, y)+p_{n+1}(x, y)+\cdots+p_{j}(x, y)+\cdots, \\
& F_{2}(x, y)=q_{n}(x, y)+q_{n+1}(x, y)+\cdots+q_{j}(x, y)+\cdots,
\end{aligned}
$$

with $n=\min \left\{\operatorname{subdeg}_{\mid(0,0)} F_{1}(x, y)\right.$, $\left.\operatorname{subdeg}_{\mid(0,0)} F_{2}(x, y)\right\} \geq 2$. We recall that given an analytic function $f(x, y)$ defined in a neighborhood of a point $\left(x_{0}, y_{0}\right)$, we define subdeg $_{\mid\left(x_{0}, y_{0}\right)} f(x, y)$ as the least positive integer $j$ such that some derivative $\left(\partial^{j} f / \partial x^{i} \partial y^{j-i}\right)\left(x_{0}, y_{0}\right)$ is not zero. We notice that this computation depends on the variables $(x, y)$ on which the function $f(x, y)$ depends, so we will explicit the variables used in each computation of subdeg. For instance, subdeg $_{\mid\left(x_{0}, y_{0}\right)} f(x, y)=0$, if and only if $f\left(x_{0}, y_{0}\right) \neq 0$. In (8), $p_{j}(x, y)$ and $q_{j}(x, y)$ denote homogeneous polynomials of $x$ and $y$ of degree $j \geq n$. It is possible that $p_{n}(x, y)$ or $q_{n}(x, y)$ be null but, by definition, not both of them can be null. The simplest case is to consider in the rescaling $\bar{p}=\bar{q}=1$. In fact, this case is equivalent to impose that system (8) has a first integral which can be expanded as a formal series in homogeneous parts.
The richness of the $\varepsilon$-method is that, using the parameter $\varepsilon$, the functions $h_{k}(x, y)$ need not be homogeneous parts and we can construct also a singular series expansion in the variables $x$ and $y$; see [23]. The method depends heavily on $h_{0}$ the first integral of the initial quasi-homogeneous system. The simpler is $h_{0}$ farther we go with the method. However, $h_{0}$ can be chosen using different scalings of variables $(x, y, t) \rightarrow$ $\left(\varepsilon^{\bar{p}} x, \varepsilon^{\bar{q}} y, \varepsilon^{\bar{r}} t\right)$ where $\varepsilon>0$ and $\bar{p}, \bar{q}$, and $\bar{r} \in \mathbb{Z}$, in such a way that $h_{0}$ will be as simple as possible. The method gives necessary conditions to have analytic integrability or a singular series expansion around a singular point and information about what is called in [23] the essential variables of a system. In the method developed in [23], the parameter $\varepsilon$ needs not be small. The parameter $\varepsilon$ may be relatively large (for instance $\varepsilon \rightarrow 1$ ). The convergence of series (7) with respect to $(x, y)$ must be analyzed in each particular case, and the convergent rate depends upon the nonlinear terms of the system (8). In the case of a resonant center, the most convenient $h_{0}$ is $x^{q} y^{p}$.

From now on we suppose that system (5) admits a formal first integral. Therefore, $h_{k}(x, y)$ are homogeneous polynomials of degree $k$ and we can take $h_{0}(x, y)=0$. In this case if we impose that series (7) be a formal first integral of system (5) we obtain at each order of homogeneity

$$
p x \frac{\partial h_{k}}{\partial x}(x, y)-q y \frac{\partial h_{k}}{\partial y}(x, y)+G_{k}(x, y)=0
$$

where $G_{k}(x, y)$ depends on the previous $h_{i}$ for $i=1, \ldots, k-1$. The solution of the partial equation (9) is

$$
h_{k}(x, y)=F\left(x^{q / p} y\right)-\int^{x} \frac{G_{k}\left(s,\left(x^{q / p} y\right) / s^{q / p}\right)}{p s} d s,
$$

where $F$ is an arbitrary function of $x^{q / p} y$. We require that $h_{k}$ be a polynomial and therefore an $h_{k}$ not having logarithmic terms. In order to avoid the logarithmic terms, it is easy to see that the function $G_{k}(x, y)$ must not have polynomial terms of the form $\left(x^{q} y^{p}\right)^{m}$ for $m \in \mathbb{N}$. This result is the same as that we obtain using normal form theory; see [6].

We must impose the vanishing of all the logarithmic terms (if there exists any difference from zero) and prove that all the functions $h_{i}(x, y)$ for $i>1$ are polynomials. In fact we must prove this by induction assuming that all the $h_{i}$ for $i=1, \ldots k-1$ are polynomials, and prove that the recursive partial differential equation with respect to $h_{k}$ (see (9)) gives also a polynomial. This is not so easy and even less working with a partial recursive differential equation as (9).

At this point we think in the blow-up $z=y / x$ because each $h_{i}(x, y)$ are homogeneous polynomials of degree $i$. 
This blowup transforms the system (5) into a system of variables $(x, z)$ of the form

$$
\begin{gathered}
\dot{z}=-(p+q) z+x \mathscr{F}(x, z), \\
\dot{x}=p x+x^{2} \mathscr{G}(x, z),
\end{gathered}
$$

where $\mathscr{F}(0,0)=0$. After that we propose a formal first integral of the form

$$
\widetilde{H}=\sum_{i \geq 1}^{\infty} f_{i}(z) x^{i}
$$

where $f_{i}(z)$ must be polynomials of degree $\leq i$ (if the logarithmic terms are zero). If we impose that series (12) be a first integral of the system in variables $(x, z)$, we obtain that the term $b_{20} x^{2}$ must be zero. However, we can vanish this term doing the change $\bar{y}=y-\alpha x^{2}$ and $\bar{x}=x$, with $\alpha=-b_{20} /(2 p+q)$. We must also take $f_{1}(z)=0$. Finally at each power of $y$ we have the recursive equation

$$
k p f_{k}(z)-(p+q) z f_{k}^{\prime}(z)+g_{k}(z)=0
$$

where $g_{k}(z)$ depends of the previous functions $f_{i}$ for $i=$ $1, \ldots, k-1$. Hence in this case instead of getting the partial recursive differential equation (9), we obtain an ordinary recursive differential equation whose solution is given by

$$
f_{k}(z)=\widetilde{C}_{k} z^{k p /(p+q)}+z^{k p /(p+q)} \int^{z} \frac{s^{-1-k p /(p+q)}}{p+q} g_{k}(s) d s
$$

where $\widetilde{C}_{k}$ is an arbitrary constant. However, we are going to see that, although we place in a resonant center where all the $f_{k}$ be polynomials, this blowup does not allow us to prove, in general, by induction of the existence of a formal first integral of the form (12).

In this sense, consider the recursive differential equation (13) whose solution is (14) and we assume that system (5) has a resonant degenerate center at the origin. It is easy to see that there always exists a value $k_{0}$ such that for $k \geq k_{0}$ the arbitrary polynomials $f_{i}(z)$ for $i=1, \ldots, k_{0}-1$ can gives following the recursive equation (13) logarithmic terms. Therefore, we can not apply the induction method to prove that the recursive equation (13) gives always a polynomial.

To see this we must to see that the solution (14) of the recursive equation (13) can give a logarithmic term. This happens when

$$
-1-\frac{k_{0} p}{p+q}+m_{k}=-1
$$

where $m_{k}$ is the degree of the polynomial $g_{k}(s)$. From here we have that $k_{0}=m_{k}(p+q) / p$. Hence if $p=1$ then $k_{0}=m_{k}(1+q)$ which can be satisfied because $m_{k}$ and $q$ are positive integers. For the case $p \neq 1$ and taking into account that the value of $m_{k}$ increases when $k$ increases, it can also exist a value of $m_{k}$ such that $m_{k}$ is divisible by $p$ and that gives the value of $k_{0}$ that can give logarithmic terms.
Therefore the conclusion is that the formal construction of the first integral (7) using homogeneous terms or using the blow-up $z=y / x$ and the formal series (12) do not allow to use the induction method in order to verify the existence of a formal first integral. We must use other developments which is the subject of the next section.

\section{Other Developments of the Formal First Integral}

In this section, we consider other developments of the formal first integral. We consider the formal development of the first integral of system (5) in a series in the variable $x$ or in $y$; that is, we consider

$$
H_{1}=\sum_{k=0}^{\infty} f_{k}(x) y^{k}, \quad \text { or } \quad H_{2}=\sum_{k=0}^{\infty} g_{k}(y) x^{k} .
$$

First we consider a general analytic system that we can always write into the following forms:

$$
\dot{x}=f(x)+y \Phi_{1}(x, y), \quad \dot{y}=g(x)+y \Phi_{2}(x, y),
$$

or

$$
\dot{x}=f(y)+x \Psi_{1}(x, y), \quad \dot{y}=g(y)+x \Psi_{2}(x, y),
$$

where $f, g, \Phi_{1}, \Phi_{2}, \Psi_{1}$, and $\Psi_{2}$ are analytic in their variables. For systems (17) and (18), we have the following straightforward result.

Proposition 4. If we impose that the series $H_{1}\left(H_{2}\right.$, resp.) be a first integral of system (17) (system (18), resp.) among others, the first condition is $f_{0}^{\prime}(x) f(x)+f_{1}(x) g(x)=0,\left(f_{0}^{\prime}(y) g(y)+\right.$ $f_{1}(y) f(y)=0$, resp.).

In order that this condition generates a collection of recursive differential equations where each $f_{i}(x)$ does not depend on $f_{i+1}(x)$, we must impose $g(x)=0(f(y)=0$, resp. $)$ which implies that $y=0(x=0$ resp. $)$ is an invariant algebraic curve of system (17) (system (18) resp.).

In fact for system (5), there are always a new coordinates $\left(z_{1}, z_{2}\right)$ where $z_{1}=0$ and $z_{2}=0$ are invariant curves. These invariant curves are defined by the stable and instable manifold of the $p:-q$ resonant singular point and therefore the previous conditions are directly satisfied. In these coordinates any $p:-q$ resonant singular point is a Lotka-Volterra system. In the following result, we are going to see that we always can find a new coordinate where $y=0$ is an invariant algebraic curve of the transformed system (5).

We consider system (2) which we write into the form

$$
\begin{gathered}
\dot{x}=P(x, y)=p x\left(1+P_{0}(x)\right)+\sum_{j \geq 1} P_{j}(x) y^{j}, \\
\dot{y}=Q(x, y)=x Q_{0}(x)-q y\left(1+Q_{1}(x)\right)+\sum_{j \geq 2} Q_{j}(x) y^{j},
\end{gathered}
$$

where $P_{j}(x)=\sum_{i \geq 0} a_{i j} x^{i}$ and $Q_{j}(x)=\sum_{i \geq 0} b_{i j} x^{i}$ are analytic functions, defined in a neighborhood of the origin. 
Lemma 5. System (19) is orbitally equivalent to

$$
\begin{gathered}
\dot{x}=P(x, y)=p x\left(1+P_{0}(x)\right)+\sum_{j \geq 1} P_{j}(x) y^{j}, \\
\dot{y}=Q(x, y)=-q y+\sum_{j \geq 2} Q_{j}(x) y^{j},
\end{gathered}
$$

that is, $Q_{0}(x) \equiv Q_{1}(x) \equiv 0$. Moreover, $(p, q)$ are coprimes.

Proof. First we prove that we can achieve $Q_{0}(x) \equiv 0$. Assume that $Q_{0}(x) \not \equiv 0$ and let $N=\min \left\{i \in \mathbb{N} \cup\{0\}: b_{i 0} \neq 0\right\}$. The change of variable $u=x, v=y-\left(b_{N 0} /(p(N+1)+q)\right) x^{N+1}$ transforms system (19) into

$$
\begin{gathered}
\dot{u}=p u\left(1+\widetilde{P}_{0}(u)\right)+\sum_{j \geq 1} \widetilde{P}_{j}(u) v^{j}, \\
\dot{v}=u \widetilde{Q}_{0}(u)-q v\left(1+\widetilde{Q}_{1}(u)\right)+\sum_{j \geq 2} \widetilde{Q}_{j}(u) v^{j},
\end{gathered}
$$

where $\widetilde{Q}_{0}(u)=\sum_{i \geq 0} \widetilde{b}_{i 0} u^{i}$ and $\widetilde{b}_{i 0}=0$ for $i=0,1, \ldots, N$ because $\dot{v}=\dot{y}-\left((N+1) b_{N 0} /(p(N+1)+q)\right) u^{N} \dot{x}$ hence

$$
\begin{aligned}
u \widetilde{Q}_{0}(u)= & b_{N 0} u^{N+1}-\frac{q b_{N 0}}{p(N+1)+q} u^{N+1} \\
& -\frac{p(N+1) b_{N 0}}{p(N+1)+q} u^{N+1}+\mathcal{O}\left(u^{N+2}\right) \\
= & \mathcal{O}\left(u^{N+2}\right) .
\end{aligned}
$$

Therefore, by means of successive change of variables we can transform system (19) into

$$
\begin{gathered}
\dot{u}=p u\left(1+\widehat{P}_{0}(u)\right)+\sum_{j \geq 1} \widehat{P}_{j}(u) y^{j}, \\
\dot{v}=-q v\left(1+\widehat{Q}_{1}(u)\right)+\sum_{j \geq 2} \widehat{Q}_{j}(u) v^{j} .
\end{gathered}
$$

To complete the proof it is enough to apply the scaling $d t=$ $d T /\left(1+\widehat{Q}_{1}(u)\right)$.

Lemma 6. Let $\mathscr{G}$ be a formal function with $\mathscr{G}(0)=0$. The system

$$
\left(\begin{array}{c}
\dot{x} \\
\dot{y}
\end{array}\right)=\left(\begin{array}{c}
p x \\
-q y
\end{array}\right)+\left(\begin{array}{c}
p x \\
q y
\end{array}\right) \mathscr{G}\left(x^{q} y^{p}\right),
$$

is formally integrable if and only if $\mathscr{G} \equiv 0$.

Proof. The sufficient condition is trivial since $x^{q} y^{p}$ is a first integral of $(\dot{x}, \dot{y})^{T}=(p x,-q y)^{T}$.

We now will prove the necessary condition. Let $\mathbf{F}(x, y)=$ $(p x,-q y)^{T}+(p x, q y)^{T} \mathscr{G}(h)$, where $h=x^{q} y^{p}$ and $\mathscr{G}(h)=$ $\sum_{j \geq 1} g_{j} h^{j} . \mathscr{G} \not \equiv 0$; otherwise, the proof is finished. We consider $j_{0}=\min \left\{j \in \mathrm{N} \mid g_{j} \neq 0\right\}$. If $I$ is a first integral of this system, then there exists $M \in \mathbb{N}$ such that $I=h^{M}+\cdots$ and $0=\nabla I \cdot \mathbf{F}=2 p q g_{j_{0}} h^{j_{0}+1}+\cdots$. Therefore, $g_{j_{0}}=0$ and this is a contradiction.
The next result shows that any integrable system (20) admits always a first integral of the form $H_{1}$.

Proposition 7. If system (20) is formally integrable then $H(x, y)=\sum_{j \geq 0} f_{j}(x) y^{j}$ with $f_{j}(x)$ a formal function is a first integral and moreover $f_{0}=\cdots f_{p-1}=0$.

Proof. By applying the Poincare-Dulac normal form, there exists a change of variable to transform (20) into

$$
\left(\begin{array}{c}
\dot{u} \\
\dot{v}
\end{array}\right)=\left(\begin{array}{c}
p u \\
-q v
\end{array}\right)+\left(\begin{array}{c}
\sum_{k \geq 1} a_{k} u h^{k} \\
\sum_{k \geq 1} b_{k} v h^{k}
\end{array}\right),
$$

where $h=u^{q} v^{p}$. We can assume that the change of variable is $u=x+\cdots, v=y(1+\cdots)$ because the axis $y=0$ and $v=0$ are invariant.

Considering the formal functions $\mathscr{F}(h)=\sum_{k \geq 1} c_{k} h^{k}$ and $\widetilde{\mathscr{G}}(h)=\sum_{k \geq 1} d_{k} h^{k}$ with $c_{k}=\left(q a_{k}-p b_{k}\right) / 2 p q$ and $d_{k}=\left(q a_{k}+\right.$ $\left.p b_{k}\right) / 2 p q$, we get

$$
\left(\begin{array}{c}
\dot{u} \\
\dot{v}
\end{array}\right)=\left(\begin{array}{c}
p u \\
-q v
\end{array}\right)\left(1+\mathscr{F}\left(u^{q} v^{p}\right)\right)+\left(\begin{array}{c}
p u \\
q v
\end{array}\right) \widetilde{\mathscr{G}}\left(u^{q} v^{p}\right),
$$

where $\mathscr{F}, \widetilde{\mathscr{G}}$ are formal functions with $\mathscr{F}(0)=\widetilde{\mathscr{G}}(0)=0$.

Moreover, by the scaling of the time, $d t=(1+\mathscr{F}) d \tau$, we can get $\mathscr{F} \equiv 0$; that is, it is possible to transform (20) into

$$
\left(\begin{array}{c}
u^{\prime} \\
v^{\prime}
\end{array}\right)=\left(\begin{array}{c}
p u \\
-q v
\end{array}\right)+\left(\begin{array}{c}
p u \\
q v
\end{array}\right) \mathscr{G}\left(u^{q} v^{p}\right),
$$

where $\mathscr{G}=\widetilde{\mathscr{G}} /(1+\mathscr{F})$ is a formal function with $\mathscr{G}(0)=0$.

If this system is formally integrable, applying Lemma 6 , we obtain $\mathscr{G}=0$. Therefore, $H(x, y)=(x+\cdots)^{q} y^{p}(1+\cdots)^{p}=$ $\sum_{k \geq p} f_{k}(x) y^{k}$ is a first integral of system (20).

The main result of this work is as follows.

Theorem 8. System (20) admits an analytic first integral $H(x, y)=\sum_{k \geq p} f_{k}(x) y^{k}$ if for each $k \in \mathbb{N}$ such that $k=M p$ with $M \in \mathbb{N}$ is verified that the derivative $\widehat{g}_{k}^{(M q)}(0)=0$, where $\widehat{g}_{k}$ for $k \geq p$ is the analytical function

$$
\begin{gathered}
\hat{g}_{k}(x):=\frac{g_{k}(x)}{p\left(1+P_{0}(x)\right) \bar{f}_{p}(x)^{k}}, \\
\bar{f}_{p}(x)=\exp \left(-\int_{0}^{x}\left(\frac{q P_{0}(s)}{p s\left(1+P_{0}(s)\right)}\right) d s\right), \\
g_{p}=0, \quad \text { and for } k>p \\
g_{k}(x)=\sum_{j=1}^{k-p}\left((k-j) f_{k-j}(x) Q_{j+1}(x)+f_{k-j}^{\prime}(x) P_{j}(x)\right),
\end{gathered}
$$

and for $k \geq p$

$$
f_{k}(x)=x^{k q / p} \bar{f}_{p}(x)^{k}\left(C_{k}-\int^{x} s^{-1-(k q / p)} \widehat{g}_{k}(s) d s\right) .
$$


Proof. By Proposition 7, we have $f_{0}=\cdots=f_{p-1}=0$. If we impose that $H(x, y)$ be a first integral of system (20), we obtain that the first condition is

$$
-p q f_{p}(x)+p x f_{p}^{\prime}(x)\left(1+P_{0}(x)\right)=0 .
$$

The next coefficients for each power of $y$ are of the form

$$
-q k f_{k}(x)+p x\left(1+P_{0}(x)\right) f_{k}^{\prime}(x)+g_{k}(x)=0,
$$

where $g_{p}=0$ and $g_{k}(x)$ for $k>p$ depends of the previous $f_{i}(x)$ for $i=p, p+1, \ldots, k-1$. More specifically we obtain the expression (28). is

The solution of homogeneous equation associated to (31)

$$
f_{k}^{h}(x)=\exp \left(\int \frac{k q}{p x\left(1+P_{0}(x)\right)}\right) .
$$

We can define the following analytical function $b(x)=$ $-(q / p x)\left(P_{0}(x) /\left(1+P_{0}(x)\right)\right)$ and the integrand of the previous expression of $f_{k}^{h}$ is a rational function which admits the following fraction decomposition:

$$
\frac{k q}{p x\left(1+P_{0}(x)\right)}=\frac{k q}{p x}+k b(x) .
$$

Therefore, $f_{k}^{h}(x)=x^{k q / p} \exp \left(\int k b(x)\right)=$ $x^{k q / p}\left(\exp \left(\int_{0}^{x} b(s) d s\right)\right)^{k}$ and for the first equation which corresponds to $k=p$ we have that $g_{p}(x)=0$. Therefore, $f_{p}^{h}(x)$ has the form $f_{p}^{h}(x)=C_{p} x^{q}\left(\bar{f}_{p}(x)\right)^{p}$ where $\bar{f}_{p}(x)=$ $\exp \left(\int_{0}^{x} b(s) d s\right)$ is an analytic function with $\bar{f}_{p}(0)=1$. So, $f_{k}^{h}(x)=C_{k} x^{k q / p} \bar{f}_{p}(x)^{k}$ and the solution of (31) is given by

$$
f_{k}(x)=x^{k q / p} \bar{f}_{p}(x)^{k}\left(C_{k}-\int_{0}^{x} \frac{s^{-k q / p} \bar{f}_{p}(s)^{-k}}{p s\left(1+P_{0}(s)\right)} g_{k}(s) d s\right) .
$$

Taking into account the form of $g_{k}(x)$, we obtain the expression (29) for $f_{k}$. Now it is straightforward to see that the integral does not give logarithmic terms in the case $k q / p \notin \mathbb{N}$ and in the case $k=M p$ because $\widehat{g}_{k}^{(M q)}(0)=0$. If we choose $C_{k}=0$, we have that each $f_{k}(x)$ for all $k$ is an analytic function and therefore system (20) under the assumptions of the theorem admits a formal first integral of the form $H(x, y)=\sum_{k \geq p} f_{k}(x) y^{k}$.

Corollary 9. System (20) admits a formal first integral of the form $H(x, y)=\sum_{j \geq p} f_{j}(x) y^{j}$ if one of the following conditions holds:

(a) $P_{0}(x) \equiv 0$ and for each $k=M p, M \in \mathbb{N}, g_{k}(x)$ is a polynomial of degree at most $M q-1$;

(b) $P_{0}(x) \equiv a x^{r}$, with $r$ a positive integer and for each $k=M p, M \in \mathbb{N}, g_{k}(x)$ is a rational function of the form $g_{k}(x)=\widetilde{g}_{k}(x) /\left(1+a x^{r}\right)^{-1+q / r p}$ where $\tilde{g}_{k}$ is a polynomial of degree at most $M q-1$.
Proof. In case (a) it is enough to apply Theorem 8 for $\bar{f}_{p}(x) \equiv$ 1 and $\hat{g}_{k}(x)=g_{k}(x) / p$. For $k=M p, M \in \mathbb{N}$ and we have $\hat{g}_{k}^{(M q)}(x)=g_{k}^{(M q)} / p=0$, because the degree of $g_{k}(x)$ is less than $q M$.

In case (b), we have that $\bar{f}_{p}(x) \equiv\left(1+a x^{r}\right)^{-q / r p}$, and $\widehat{g}_{k}(x)=g_{k}(x) / p\left(1+a x^{r}\right)^{1-q / r p}$. Therefore, $\widehat{g}_{k}(x)=\widetilde{g}_{k}(x) / p$ and $\hat{g}_{k}^{(M p)}(x)=0$ because the degree of $\widetilde{g}_{k}(x)$ is less than $q M$. We obtain the result by applying also Theorem 8 .

Notice that in fact statement (a) is contained in statement (b). However, we give the two statements in a separate form in order to be applied directly to different examples.

Lemma 10. If system (20) with $P_{0}(x) \equiv 0$ admits a formal first integral of the form $H(x, y)=\sum_{j \geq p} f_{j}(x) y^{j}$ then the following conditions hold.

(a) If for each $k$ such that $k q / p \notin \mathbb{N}, g_{k}$ is a polynomial then it is possible to choose $f_{k}$ polynomial with $\operatorname{deg}\left(f_{k}\right)=\operatorname{deg}\left(g_{k}\right)$.

(b) If for each $k=M p, M \in \mathbb{N}, g_{k}(x)$ is a polynomial with $\operatorname{deg}\left(g_{k}\right) \leq M q-1$ then it is possible to choose $f_{k}$ polynomial with $\operatorname{deg}\left(f_{k}\right) \leq M q-1$.

Proof. By applying Theorem 8 , we obtain $\bar{f}_{p}(x) \equiv 1$ and $\widehat{g}_{k}(x)=g_{k}(x) / p$. If we choose $C_{k}=0$ in (29), we obtain

$$
f_{k}(x)=-\frac{1}{p} x^{k q / p} \int^{x} s^{-1-k q / p} g_{k}(s) d s .
$$

In case (a), the result is trivial since the integral does not give logarithmic terms because $k q / p \notin \mathbb{N}$.

In case (b), $k=M p$ and $f_{k}(x)=$ $-(1 / p) x^{M q} \int^{x} s^{-1-M q} g_{k}(s) d s$. Therefore, we obtain the result applying that $g_{k}$ is a polynomial with $\operatorname{deg}\left(g_{k}\right) \leq M q-1$.

Based on the results presented in this work, the following proposition gives a large family of analytic systems that have an analytic first integral.

Proposition 11. The analytic system

$$
\begin{gathered}
\dot{x}=p x+\sum_{j \geq 1} P_{j}(x) y^{j}, \\
\dot{x}=-q y+\sum_{j \geq 2} Q_{j}(x) y^{j},
\end{gathered}
$$

where $P_{j}$ and $Q_{j}$ are polynomials such that $\operatorname{deg}\left(P_{j}\right) \leq\lfloor j q / p\rfloor$, $\operatorname{deg}\left(Q_{j}\right) \leq\lfloor(j-1) q / p\rfloor-1$ has an analytic first integral in a neighborhood of the origin where $\lfloor a\rfloor$ is the integer part of $a \in \mathbb{R}$.

Proof. From Proposition 7, if system (36) is integrable then a first integral is of the form $H(x, y)=\sum_{j \geq p} f_{j}(x) y^{j}$ where $f_{j}(x)$ is a formal function. Moreover, $P_{0}(x) \equiv 0$ and, therefore, by applying Theorem 8 we obtain $\bar{f}_{p}(x) \equiv 1$, $\widehat{g}_{k}(x)=g_{k}(x) / p, g_{p}=0$ and $f_{p}(x)=x^{q}$. 
We will prove now by induction that $\operatorname{deg}\left(f_{j}\right) \leq\lfloor j q / p\rfloor$ and $\operatorname{deg}\left(g_{j}\right) \leq\lfloor j q / p\rfloor-1$ for all $j \in \mathbb{N}$ and $p \leq j$.

For $j=p$ is true since $f_{p}(x)=x^{q}$ and $g_{p}=0$. If we suppose that the hypothesis is true for $1 \leq j \leq j_{0}-1$, we have to prove that $\operatorname{deg}\left(g_{j_{0}}\right) \leq\left\lfloor j_{0} q / p\right\rfloor-1$ and $\operatorname{deg}\left(f_{j_{0}}\right) \leq\left\lfloor j_{0} q / p\right\rfloor$.

On the other hand, applying (28), we have

$$
\begin{aligned}
& \operatorname{deg}\left(g_{j_{0}}\right) \\
& \leq \max _{1 \leq j \leq j_{0}-p}\left\{\operatorname{deg}\left(f_{j_{0}-j}\right)+\operatorname{deg}\left(Q_{j+1}\right), \operatorname{deg}\left(f_{j_{0}-j}^{\prime}\right)+\operatorname{deg}\left(P_{j}\right)\right\} \\
& \leq\left\lfloor\frac{j_{0} q}{p}\right\rfloor-1,
\end{aligned}
$$

since for $1 \leq j \leq j_{0}-p$, we have

$$
\begin{aligned}
\operatorname{deg}\left(f_{j_{0}-j}\right)+\operatorname{deg}\left(Q_{j+1}\right) & \leq\left\lfloor\left(j_{0}-j\right) q / p\right\rfloor+\left\lfloor\frac{j q}{p}\right\rfloor-1 \\
& \leq\left\lfloor\frac{j_{0} q}{p}\right\rfloor-1, \\
\operatorname{deg}\left(f_{j_{0}-j}^{\prime}\right)+\operatorname{deg}\left(P_{j}\right) & \leq\left\lfloor\frac{\left(j_{0}-j\right) q}{p}\right\rfloor-1+\left\lfloor\frac{j q}{p}\right\rfloor \\
& \leq\left\lfloor\frac{j_{0} q}{p}\right\rfloor-1 .
\end{aligned}
$$

By applying Lemma 10, we have that $\operatorname{deg}\left(f_{j_{0}}\right) \leq\left\lfloor j_{0} q / p\right\rfloor-$ 1. In particular, $\operatorname{deg}\left(f_{j_{0}}\right) \leq\left\lfloor j_{0} q / p\right\rfloor$.

Therefore, if $k=M p, \operatorname{deg}\left(g_{k}\right) \leq M q-1, g_{k}^{M q}(0)=0$ and $\hat{g}_{k}^{M q}(0)=0$.

To finish the proof, it is enough to apply Theorem 8.

However, as it is difficult to determine the new coordinates where system (19) has $Q_{0}(x) \equiv Q_{1}(x) \equiv 0$, we are going to work with the original system (5), imposing directly these two conditions. In order that one of the series $H_{1}$ or $\mathrm{H}_{2}$ be a formal first integral of system (5), we have that these series must have the term $x^{q} y^{p}$ as a first monomial in its development. We will see that in this case and under some conditions we can use the induction method in order to verify the existence of a formal first integral.

The following result is only established for the series $H_{1}$ but we can obtain a similar result for the case where $\mathrm{H}_{2}$ is a formal first integral of system (5).

Proposition 12. We consider system (5) where we write $F_{1}$ and $F_{2}$ into the form

$$
\begin{aligned}
& F_{1}(x, y)=\sum_{i+j=2}^{\infty} a_{i j} x^{i} y^{j}, \\
& F_{2}(x, y)=\sum_{i+j=2}^{\infty} b_{i j} x^{i} y^{j},
\end{aligned}
$$

where $a_{i j}$ and $b_{i j}$ are arbitrary constants. If we impose that $H_{1}$ be a first integral of system (5), we obtain that the first condition is

$$
\sum_{i=2}^{\infty} b_{i 0} x^{i} f_{1}(x)+p x f_{0}^{\prime}(x)+\sum_{i=2}^{\infty} a_{i 0} x^{i} f_{0}^{\prime}(x)=0 .
$$

The proof of this proposition is also straightforward developing in powers of $y$ and taking the coefficient of the power $y^{0}$. Hence in order to have a recursive differential equations where each $f_{i}(x)$ does not depend on $f_{i+1}(x)$, we must impose that $b_{i 0}=0$ for all $i$ and consequently $f_{0}$ must be a constant that we can take without loss of generality equal to zero. The consequence, as before, is that if all $b_{i 0}=0$ for all $i$ the system (5) has $y$ as invariant algebraic curve.

The next coefficients for each power of $y$ are of the form

$$
\begin{aligned}
k(-q & \left.+\sum_{i=1}^{\infty} b_{i 1} x^{i}\right) f_{k}(x) \\
+ & \left(p x+\sum_{i=2}^{\infty} a_{i 0} x^{i}\right) f_{k}^{\prime}(x)+g_{k}(x)=0,
\end{aligned}
$$

where $g_{k}(x)$ depends of the previous $f_{i}(x)$ for $i=1,2, \ldots, k-$ 1 , and for the first equation which corresponds to $k=1$ we have that $g_{1}(x)=0$. Therefore, $f_{1}(x)$ has the form

$$
f_{1}(x)=\exp \left(-\int \frac{-q+\sum_{i=1}^{\infty} b_{i 1} x^{i}}{p x+\sum_{i=2}^{\infty} a_{i 0} x^{i}}\right) .
$$

The integrand of the previous expression of $f_{1}$ is a rational function which admits the following fraction decomposition:

$$
\frac{-q+\sum_{i=1}^{\infty} b_{i 1} x^{i}}{p x+\sum_{i=2}^{\infty} a_{i 0} x^{i}}=\frac{A}{x}+\frac{B(x)}{p+\sum_{i=2}^{\infty} a_{i 0} x^{i-1}},
$$

where $A=-q / p$ and $B(x)$ is a formal series. Therefore, $f_{1}(x)=x^{q / p} \bar{f}_{1}(x)$ where $\bar{f}_{1}$ is the corresponding formal series obtained after the integration. Now we consider the particular case $p=1$. The following theorem gives sufficient conditions to have formal integrability in this case. A similar proposition can be established for the case when the first integral is of the form $\mathrm{H}_{2}$.

Proposition 13. System (5) where we write $F_{1}$ and $F_{2}$ into the form (39) admits a formal first integral of the form $H_{1}$ if $p=1$, $b_{i 0}=0$ for $i \geq 2$ and $b_{i 1}=0$ for $i \geq 1$ and

$$
g_{k}(x)=\frac{\tilde{g}_{k}(x) P(x, 0)}{x \bar{f}_{1}(x)^{-k}} \text { with } \tilde{g}_{k}^{(k q)}(0)=0 .
$$

Proof. Under the assumptions of the theorem, we have that $f_{0}=0, f_{1}=x^{q} \bar{f}_{1}(x)$, and (41) takes the form

$$
-k q f_{k}(x)+P(x, 0) f_{k}^{\prime}(x)+g_{k}(x)=0,
$$

whose solution is given by

$$
f_{k}(x)=x^{k q} \bar{f}_{1}(x)^{k}\left(C_{k}-\int^{x} \frac{s^{-k q} \bar{f}_{1}(s)^{-k}}{P(s, 0)} g_{k}(s) d s\right) .
$$


Taking into account the form of $g_{k}(x)$, we obtain

$$
f_{k}(x)=x^{k q} \bar{f}_{1}(x)^{k}\left(C_{k}-\int^{x} s^{-1-k q} \widetilde{g}_{k}(s) d s\right) .
$$

Now it is straightforward to see that the integral does not give logarithmic terms because the $k q$-th derivative of $\widetilde{g}_{k}(s)$ at zero is zero; that is, $\widetilde{g}_{k}^{(k q)}(0)=0$. We have that each $f_{k}(x)$ for all $k$ is an analytic function and therefore system (5) under the assumptions of the theorem admits a formal first integral of the form (39).

Examples where Proposition 13 can be applied can be found in $[7,12,16,17]$ where some partial results are given. We also can establish the following corollary in the case where the $g_{k}(x)$ are polynomials or some irrational functions.

Corollary 14. System (5) where we write $F_{1}$ and $F_{2}$ into the form (39) admits a formal first integral of the form $\mathrm{H}_{1}$ if one of the following conditions holds:

(a) $p=1, a_{i 0}=b_{i 0}=0$ for $i \geq 2$ and $b_{i 1}=0$ for $i \geq 1$ and $g_{k}(x)$ is a polynomial of degree at most $k q-1$;

(b) $p=1, a_{i 0}=0$ for $i \neq r+1, b_{i 0}=0$ for $i \geq 2$ and $b_{i 1}=0$ for $i \geq 1$ with $r$ a positive integer and $g_{k}(x)$ is a rational function of the form $g_{k}(x)=\tilde{g}_{k}(x) /\left(1+a x^{r}\right)^{-1+k q / r}$ where $\widetilde{g}_{k}$ is a polynomial of degree at most $k q-1$.

Proof. In case (a) for we have $f_{0}=0$ and $f_{1}=x^{q}$ (41) take the form

$$
-k q f_{k}(x)+x f_{k}^{\prime}(x)+g_{k}(x)=0,
$$

whose solution is given by

$$
f_{k}(x)=C_{k} x^{k q}-x^{k q} \int^{x} s^{-1-k q} g_{k}(s) d s,
$$

and taking into account that $g_{k}(x)$, that depends on $f_{i}$ for $i=$ $1,2, \ldots, k-1$, is at most of degree $k q-1$, we obtain that $f_{k}$ is a polynomial. Moreover, in this case system (5) becomes

$$
\begin{gathered}
\dot{x}=p x+y \phi_{1}(x, y), \\
\dot{y}=-q y+y \phi_{2}(x, y),
\end{gathered}
$$

where $\phi_{1}$ and $\phi_{2}$ are analytic functions.

In case (b), we have that $f_{0}=0, f_{1}=x^{q}\left(1+a x^{r}\right)^{-q / r}$ and (41) takes the form

$$
-k q f_{k}(x)+x f_{k}^{\prime}(x)+a x^{r+1} f_{k}^{\prime}(x)+g_{k}(x)=0,
$$

whose solution is given by

$$
\begin{aligned}
f_{k}(x)= & x^{k q}\left(1+a x^{r}\right)^{-k q / r} \\
& \times\left(C_{k}-\int^{x} s^{-1-k q}(1+a s)^{-1+k q / r} g_{k}(s) d s\right),
\end{aligned}
$$

and taking into account the form of $g_{k}(x)$, we obtain that $f_{k}(x)$ is also of the form $f_{k}(x)=p_{k}(x) /\left(1+a x^{r}\right)^{-k q / s}$ where $p_{k}(x)$ is a polynomial because the integral in (52) does not give logarithmic terms.
Notice that in fact statement (a) is contained in statement (b). However, we give the two statements in a separate form in order to be applied directly to different examples. Corollary 14 generalizes a lot of cases obtained in the literature when some concrete families of polynomials systems were studied. We have not made an exhaustive study of all the cases included in these large families but for instance contains the cases (3) and (4) of Theorem 3 in [17] and case (3) of Theorem 2 in [16].

In resume, when we study a family that satisfies the necessary conditions, we must impose if the family system has a formal first integral of the form $\mathrm{H}_{1}$ or $\mathrm{H}_{2}$. If none of them work, we can look for the proper coordinates $\left(z_{1}, z_{2}\right)$ defined by the stable and instable manifold of the $p:-q$ resonant singular point. In this way, we must study if at least one of the separatrices of the resonant point is algebraic. The best case is when both are algebraic. Therefore, the problem to find proper coordinates $\left(z_{1}, z_{2}\right)$ becomes a problem of finding the invariant algebraic curves of system (5) passing through the $p:-q$ resonant singular point. The existence of invariant algebraic curves takes a leading role in the formal integrability theory and is the base to find the proper coordinates system. This is shown in the examples provided in [12] that are solved by ad hoc methods and where the changes of variables proposed to solve the examples are always invariant algebraic curves of the original system passing through the origin. In fact, in [12], it recalled the Abhyankar-Moch theorem [37]. This theorem establishes, assuming the existence of an invariant rational algebraic curve passing through the origin, the existence of a rational invertible change of variables such that the invariant curve becomes one of the invariant axes. In this paper, this result is generalized in the sense that any $p:-q$ resonant singular point is formal orbitally equivalent to a system with $y=0$ as invariant algebraic curve; see Lemma 5.

\section{Examples}

In this section, we present some examples where the results developed in this paper are applied.

Example 15. We first consider the 1: -1 resonant quadratic system given by

$$
\begin{aligned}
& \dot{x}=x+a_{20} x^{2}+a_{11} x y+a_{02} y^{2}, \\
& \dot{y}=-y+b_{20} x^{2}+b_{11} x y+b_{02} y^{2} .
\end{aligned}
$$

The aim is not to do an exhaustive study of when system (53) has a formal first integral in a neighborhood of the origin. In fact, the complex center cases were studied by Dulac; see [34]. The aim is only to show how to use the results given in this paper. If we apply directly Corollary 14 statement (a), we obtain $b_{20}=0$ (this condition implies that $y=0$ be an algebraic invariant curve of system (53)) and $a_{20}=b_{11}=0$. In this case, the quadratic system (53) reads

$$
\begin{gathered}
\dot{x}=x+a_{11} x y+a_{02} y^{2}, \\
\dot{y}=-y+b_{02} y^{2}
\end{gathered}
$$


and admits a formal first integral, and by Theorem 2 system (54) has an analytic first integral in a neighborhood of the origin.

In fact, the associated equation to system (54) is a linear equation with respect to $x$ and the system has a Darboux first integral of the form

$$
\begin{aligned}
& H(x, y) \\
& =\left(b_{02} y-1\right)^{-1-\left(a_{11} / b_{02}\right)} \\
& \quad \times\left[2 a_{02}-2\left(a_{02} a_{11}+a_{02} b_{02}\right) y\right. \\
& \left.\quad+\left(a_{11}^{3}-a_{11} b_{02}^{2}\right) x y+\left(a_{02} a_{11}^{2}+a_{02} a_{11} b_{02}\right) y^{2}\right] .
\end{aligned}
$$

Example 16. Consider the 1:-1 resonant Lotka-Volterra planar complex quartic system given by

$$
\begin{aligned}
\dot{x}=x & \left(1-a_{10} x-a_{01} y-a_{20} x^{2}-a_{11} x y\right. \\
& \left.-a_{02} y^{2}-a_{30} x^{3}-a_{21} x^{2} y-a_{12} x y^{2}-a_{03} y^{3}\right), \\
\dot{y}=-y & \left(1-b_{10} x-b_{01} y-b_{20} x^{2}-b_{11} x y\right. \\
& \left.\quad b_{02} y^{2}-b_{30} x^{3}-b_{21} x^{2} y-b_{12} x y^{2}-b_{03} y^{3}\right) .
\end{aligned}
$$

The Lotka-Volterra planar complex systems have been studied in several papers; see [7, 11, 13-15]. In any case, the LotkaVolterra planar complex quartic system is an open problem computationally infeasible. However, with the results of this paper we are going to see that we can give some sufficient conditions to have an analytic first integral without computing any resonant focus value. We propose a formal first integral of the form $H_{1}$, that is, of the form (16). The recurrence that gives $f_{i}(x)$ is given by $-i f_{i}(x)+x f_{i}^{\prime}(x)+g_{i}(x)=0$ where $g_{i}(x)$ is

$$
\begin{aligned}
& (i-3) b_{03} f_{i-3}(x)+(i-2) b_{02} f_{i-2}(x)+(i-2) b_{12} x f_{i-2}(x) \\
& \quad+(i-1) b_{01} f_{i-1}(x)+(i-1) b_{11} x f_{i-1}(x) \\
& \quad+(i-1) b_{21} x^{2} f_{i-1}(x) \\
& \quad+i b_{10} x f_{i}(x)+i b_{20} x^{2} f_{i}(x) \\
& \quad+i b_{30} x^{3} f_{i}(x)-a_{03} x f_{i-3}^{\prime}(x)-a_{02} x f_{i-2}^{\prime}(x) \\
& \quad-a_{12} x^{2} f_{i-2}^{\prime}(x)-a_{01} x f_{i-1}^{\prime}(x)-a_{11} x^{2} f_{i-1}^{\prime}(x) \\
& \quad-a_{10} x^{2} f_{i}^{\prime}(x)-a_{20} x^{3} f_{i}^{\prime}(x)-a_{30} x^{4} f_{i}^{\prime}(x) .
\end{aligned}
$$

Now we impose that $g_{i}(x)$ must be of degree $\leq i-1$. This implies that $a_{10}=a_{20}=a_{30}=b_{10}=b_{20}=b_{30}=0$ and $b_{21}=a_{21}=b_{11}=a_{11}=0$ and in this case the recurrence is $-i f_{i}(x)+x f_{i}^{\prime}(x)+g_{i}(x)=0$ where $g_{i}(x)$ is given by

$$
\begin{aligned}
& (i-3) b_{03} f_{i-3}(x)+(i-2)\left(b_{02}+b_{12} x\right) f_{i-2}(x) \\
& \quad+(i-1) b_{01} f_{i-1}(x)-a_{03} x f_{i-3}^{\prime}(x)-a_{02} x f_{i-2}^{\prime}(x) \\
& \quad-a_{12} x^{2} f_{i-2}^{\prime}(x)-a_{01} x f_{i-1}^{\prime}(x) .
\end{aligned}
$$

We obtain that its solution is given by

$$
f_{i}(x)=C_{i} x^{i}-x^{i} \int^{x} s^{-1-i} g_{i}(s) d s
$$

and taking into account that $g_{i}(x)$ is of degree $\leq i-1$, we can establish the following result.

Proposition 17. The system

$$
\begin{gathered}
\dot{x}=x\left(1-a_{01} y-a_{12} x y^{2}-a_{03} y^{3}\right), \\
\dot{y}=-y\left(1-b_{01} y-b_{02} y^{2}-b_{12} x y^{2}-b_{03} y^{3}\right)
\end{gathered}
$$

has an analytic first integral in a neighborhood of the origin.

In fact, Proposition 17 is a consequence of Proposition 11. This technique can be applied to several families of polynomial systems and in fact gives a new mechanism to obtain resonant centers and also real centers for the complex systems that have a real preimage (when this preimage exists, see e.g., [18]).

\section{Acknowledgments}

A. Algaba and C. García are supported by a MICINN/FEDER Grant no. MTM2010-20907-C02-02 and by the Consejería de Educacíon y Ciencia de la Junta de Andalucía (projects EXC/2008/FQM-872, TIC-130, and FQM-276). J. Giné is partially supported by a MICINN/FEDER Grant no. MTM201122877 and by Generalitat de Catalunya Grant no. 2009SGR 381.

\section{References}

[1] A. Algaba, E. Gamero, and C. García, "The integrability problem for a class of planar systems," Nonlinearity, vol. 22, no. 2, pp. 395-420, 2009.

[2] A. Algaba, E. Freire, E. Gamero, and C. García, "Monodromy, center-focus and integrability problems for quasi-homogeneous polynomial systems," Nonlinear Analysis: Theory, Methods \& Applications, vol. 72, no. 3-4, pp. 1726-1736, 2010.

[3] A. Algaba, N. Fuentes, and C. García, "Centers of quasihomogeneous polynomial planar systems," Nonlinear Analysis: Real World Applications, vol. 13, no. 1, pp. 419-431, 2012.

[4] A. Algaba, C. García, and M. Reyes, "Existence of an inverse integrating factor, center problem and integrability of a class of nilpotent systems," Chaos, Solitons \& Fractals, vol. 45, no. 6, pp. 869-878, 2012.

[5] J. Chavarriga, H. Giacomini, J. Giné, and J. Llibre, "On the integrability of two-dimensional flows," Journal of Differential Equations, vol. 157, no. 1, pp. 163-182, 1999.

[6] H. Żoładek, "The problem of center for resonant singular points of polynomial vector fields," Journal of Differential Equations, vol. 137, no. 1, pp. 94-118, 1997.

[7] X. Chen, J. Giné, V. G. Romanovski, and D. S. Shafer, "The 1: $-q$ resonant center problem for certain cubic Lotka-Volterra systems," Applied Mathematics and Computation, vol. 218, no. 23, pp. 11620-11633, 2012. 
[8] C. Christopher, P. Mardešić, and C. Rousseau, "Normalizable, integrable, and linearizable saddle points for complex quadratic systems in $\mathbb{C}^{2}$," Journal of Dynamical and Control Systems, vol. 9, no. 3, pp. 311-363, 2003.

[9] C. Christopher and C. Rousseau, "Normalizable, integrable and linearizable saddle points in the Lotka-Volterra system," Qualitative Theory of Dynamical Systems, vol. 5, no. 1, pp. 11-61, 2004.

[10] B. Ferčec, X. Chen, and V. G. Romanovski, "Integrability conditions for complex systems with homogeneous quintic nonlinearities," The Journal of Applied Analysis and Computation, vol. 1, no. 1, pp. 9-20, 2011.

[11] B. Fercec, J. Gine, Y. Liu, and V. G. Romanovski, "Integrability conditions for Lotka-Volterra planar complex quartic systems having homogeneous nonlinearities," Acta Applicandae Mathematicae, vol. 124, no. 1, pp. 107-122, 2013.

[12] A. Fronville, A. P. Sadovski, and H. Żołpolhk adek, "Solution of the 1: -2 resonant center problem in the quadratic case," Fundamenta Mathematicae, vol. 157, no. 2-3, pp. 191-207, 1998.

[13] J. Giné, Z. Kadyrsizova, Y. Liu, and V. G. Romanovski, "Linearizability conditions for Lotka-Volterra planar complex quartic systems having homogeneous nonlinearities," Computers \& Mathematics with Applications, vol. 61, no. 4, pp. 1190-1201, 2011.

[14] J. Giné and V. G. Romanovski, "Linearizability conditions for Lotka-Volterra planar complex cubic systems," Journal of Physics, vol. 42, no. 22, Article ID 225206, 2009.

[15] J. Giné and V. G. Romanovski, "Integrability conditions for Lotka-Volterra planar complex quintic systems," Nonlinear Analysis: Real World Applications, vol. 11, no. 3, pp. 2100-2105, 2010.

[16] Z. Hu, V. G. Romanovski, and D. S. Shafer, "1: -3 resonant centers on $\mathbb{C}^{2}$ with homogeneous cubic nonlinearities," Computers \& Mathematics with Applications, vol. 56, no. 8, pp. 1927-1940, 2008.

[17] V. G. Romanovski, X. Chen, and Z. Hu, "Linearizability of linear systems perturbed by fifth degree homogeneous polynomials," Journal of Physics, vol. 40, no. 22, pp. 5905-5919, 2007.

[18] V. G. Romanovski and D. S. Shafer, The Center and Cyclicity Problems: A Computational Algebra Approach, Birkhäuser, Boston, Mass, USA, 2009.

[19] A. Algaba, C. García, and M. Reyes, "Local bifurcation of limit cycles and integrability of a class of nilpotent systems of differential equations," Applied Mathematics and Computation, vol. 215, no. 1, pp. 314-323, 2009.

[20] A. Algaba, C. García, and M. Reyes, "Rational integrability of two-dimensional quasi-homogeneous polynomial differential systems," Nonlinear Analysis: Theory, Methods \& Applications, vol. 73, no. 5, pp. 1318-1327, 2010.

[21] A. Algaba, C. García, and M. Reyes, "Integrability of two dimensional quasi-homogeneous polynomial differential systems," The Rocky Mountain Journal of Mathematics, vol. 41, no. 1, pp. 1-22, 2011.

[22] A. Algaba, C. Garcia, and M. Reyes, "A note on analytic integrability of planar vector fields," European Journal of Applied Mathematics, vol. 23, pp. 555-562, 2012.

[23] J. Giné and X. Santallusia, "Essential variables in the integrability problem of planar vector fields," Physics Letters A, vol. 375, no. 3, pp. 291-297, 2011.

[24] S. Gravel and P. Thibault, "Integrability and linearizability of the Lotka-Volterra system with a saddle point with rational hyperbolicity ratio," Journal of Differential Equations, vol. 184, no. 1, pp. 20-47, 2002.
[25] C. Liu, G. Chen, and G. Chen, "Integrability of Lotka-Volterra type systems of degree 4," Journal of Mathematical Analysis and Applications, vol. 388, no. 2, pp. 1107-1116, 2012.

[26] C. Liu, G. Chen, and C. Li, "Integrability and linearizability of the Lotka-Volterra systems," Journal of Differential Equations, vol. 198, no. 2, pp. 301-320, 2004.

[27] J.-F. Mattei and R. Moussu, "Holonomie et intégrales premières," Annales Scientifiques de l'École Normale Supérieure, vol. 13, no. 4, pp. 469-523, 1980.

[28] J. Chavarriga, H. Giacomin, J. Giné, and J. Llibre, "Local analytic integrability for nilpotent centers," Ergodic Theory and Dynamical Systems, vol. 23, no. 2, pp. 417-428, 2003.

[29] J. Giné, "On the number of algebraically independent PoincaréLiapunov constants," Applied Mathematics and Computation, vol. 188, no. 2, pp. 1870-1877, 2007.

[30] J. Giné, "Higher order limit cycle bifurcations from nondegenerate centers," Applied Mathematics and Computation, vol. 218, no. 17, pp. 8853-8860, 2012.

[31] J. Giné and J. Mallol, "Minimum number of ideal generators for a linear center perturbed by homogeneous polynomials," Nonlinear Analysis: Theory, Methods \& Applications, vol. 71, no. 12, pp. e132-e137, 2009.

[32] M. A. Liapunov, Problème Général de la Stabilité du Mouvement, vol. 17 of Annals of Mathematics Studies, Pricenton University Press, 1947.

[33] H. Poincaré, "Mémoire sur les courbes définies par les équations différentielles," Journal de Mathematiques, vol. 37, pp. 375-422, 1881, vol. 8, pp. 251-296, 1882, Oeuvres de Henri Poincaré, vol. I, Gauthier-Villars, Paris, pp. 3-84, 1951.

[34] H. Dulac, "Détermination et intégration d'une certaine classe d'équations différentielles ayant pour point singulier un centre," Bulletin des Sciences Mathématiques, vol. 32, no. 2, pp. 230-252, 1908.

[35] A. D. Bruno, Local Methods in Nonlinear Differential Equations, Springer, Berlin, Germany, 1989.

[36] A. D. Bruno, Power Geometry in Algebraic and Differential Equations, vol. 57 of North-Holland Mathematical Library, North-Holland Publishing Co., Amsterdam, The Netherlands, 2000.

[37] S. S. Abhyankar and T. T. Moh, "Embeddings of the line in the plane," Journal für die Reine und Angewandte Mathematik, vol. 276, pp. 148-166, 1975. 


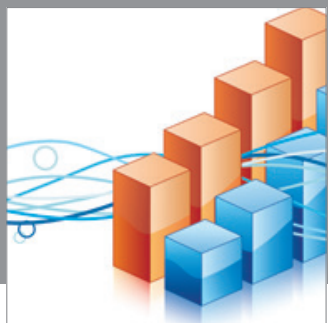

Advances in

Operations Research

mansans

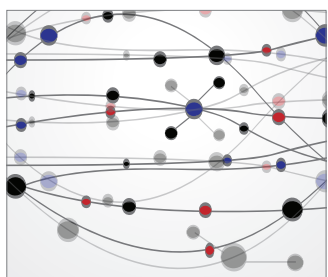

The Scientific World Journal
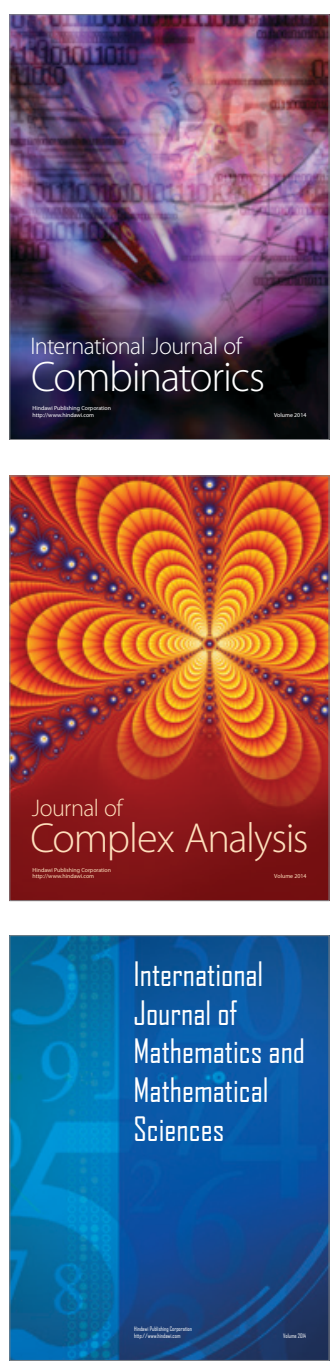
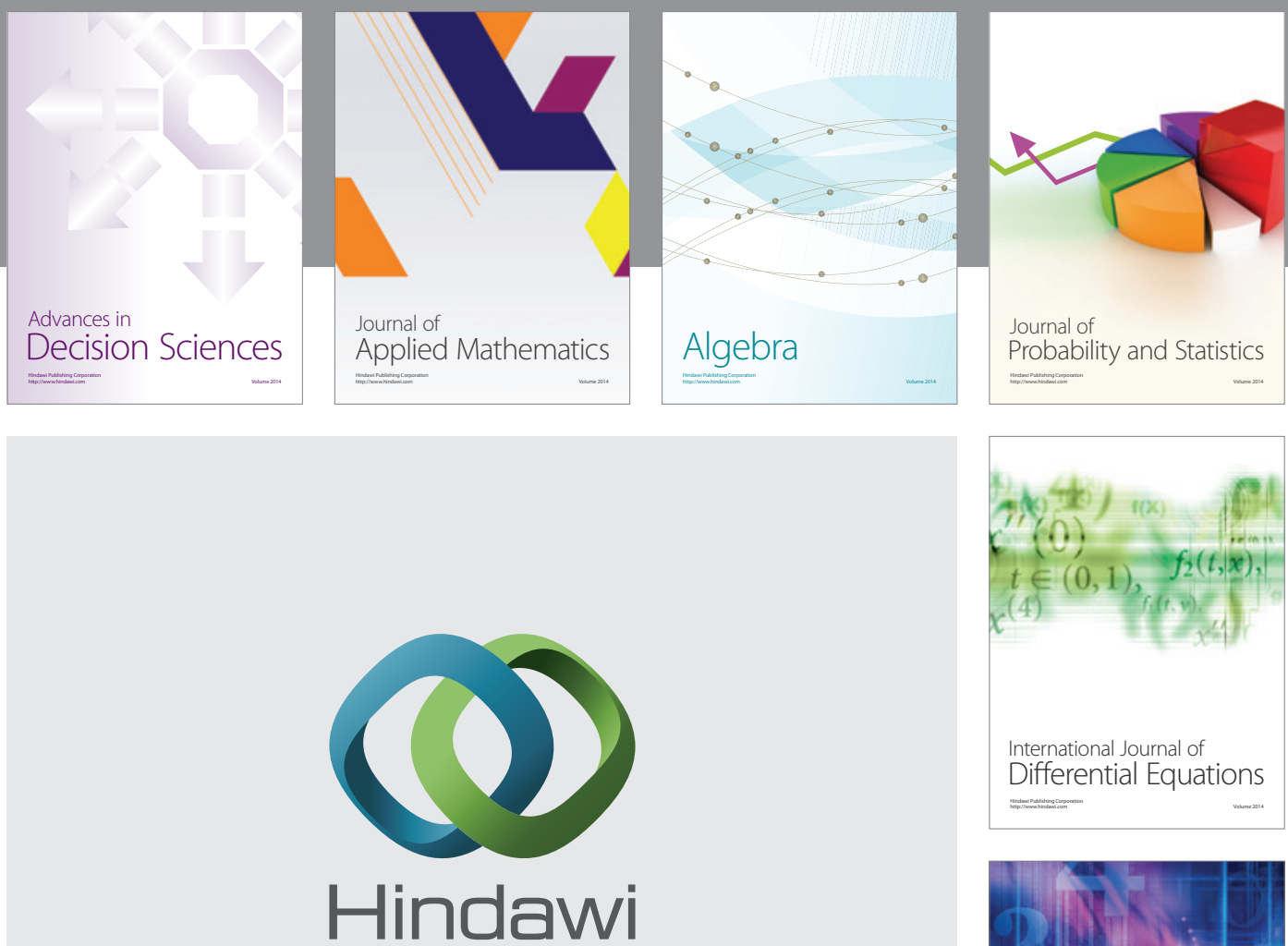

Submit your manuscripts at http://www.hindawi.com
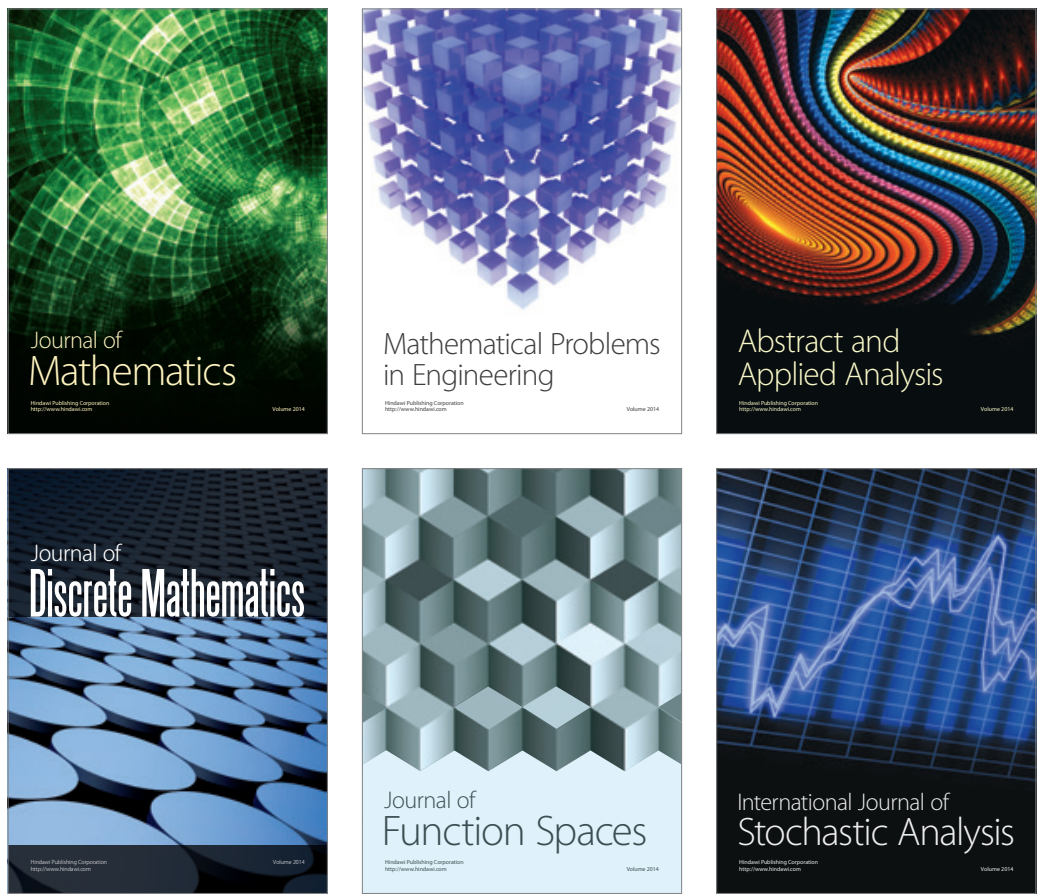

Journal of

Function Spaces

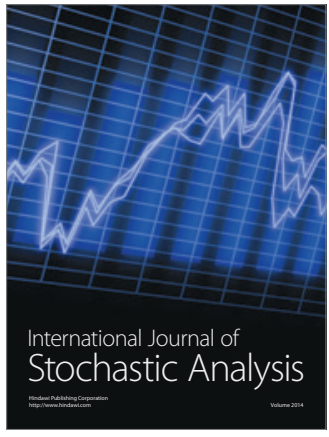

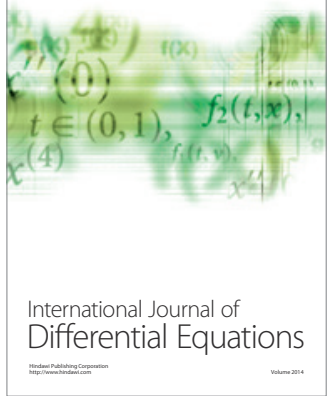
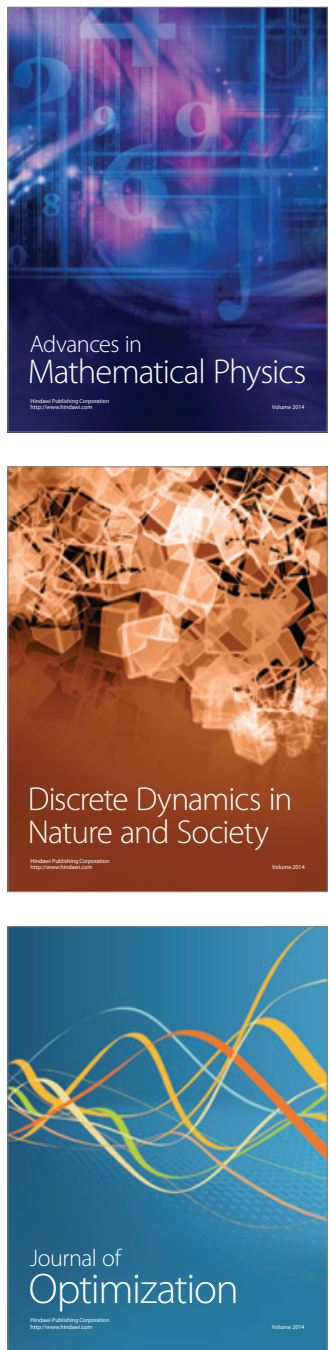